\title{
Analysis of a continuous field theory in two dimensions with use of the Density Matrix Renormalization Group
}

\author{
William Lay \\ Department of Physics, UCLA, Box 951547, Los Angeles, CA 90095-1547
}

(November 19, 2018)

\begin{abstract}
A formulation of the Ginzburg-Landau-Wilson version of the partition function of a system with a continuously varying order parameter as a transfer matrix calculation allows for the application of methods based on the Density Matrix Renormalization Group (DMRG) to the calculation of the free energy of the $O(1)$ model. The essence of both the mapping and the DMRG calculation is laid out, along with results that validate this strategy. This method forms the basis of a unified approach to the crossover from three to two dimensions in an $O(1)$ system with a slab-like geometry.
\end{abstract}

75.40.-s,05.01.-a,64.60.Ak

The formulation utilized to analyze the critical point of the $O(1)$ system in three and more dimensions differs in fundamental respects from the formulation that has proven most fruitful in two and fewer dimensions. In the former case, highly accurate results [1,2] have been obtained with the use of the Ginzburg-Landau-Wilson effective Hamiltonian, which is based on a continuously varying order parameter. On the other hand, lower dimensional systems are generally modeled with the use of a fixed-length order parameter [3,, , 1 .

The above state of affairs is acceptable, if not entirely satisfactory, as long as one is not interested in a system whose critical point properties possesses both three- and two-dimensional features. Such is the case in the instance of dimensional crossover in a system, with a slab-like geometry. Scaling relations and the Renormalization Group predict the asymptotic behavior of such a system when the correlation bulk three-dimensional correlation length is asymptotically large or small compared to the finite slab width. In addition, finite size scaling indicates the existence of a general form for the crossover between those two limits [5.6. H. However, the calculation of the specific form of the crossover function requires a unified theoretical approach to the critical point properties of a slab-like system in both the three- and two-dimensional regimes.

The calculation of the crossover function, and of the thermodynamic quantities that can be derived from it, has been carried out in a few cases. For example, crossover from three to two dimensions has been worked out in the mean spherical model [5.7] and in its mathematical equivalent, Bose-Einstein condensation. Crossover from $d$ to $d-1$ dimensions can also be determined in an $O(1)$, or Ising-like, system when $d \geq 5$ [8]. Here, both systems possess asymptotically mean-field critical behavior.

In addition to the above exact determinations of dimensional crossover, a field-theoretically motivated approach has been formulated based on the notion of an "environmentally friendly" renormalization group [9. 10]. This approach yields explicit (and relatively simple) crossover functions for thermodynamic quantities. While the functions are necessarily approximate, they are consistent with the key expectations that arise from finite-size scaling. Unfortunately, existing literature provides no simple way to improve the crossover functions that arise from the environmentally friendly renormalization group, so as to produce predictions that can be profitably compared with experimental data.

We have developed an approach that holds the promise of producing just such an improvement. This approach is based on a reformulation of the partition function of a lattice based system with a continuous order parameter in terms of transfer matrices which allows for the calculation of the partition function either with the use of field-theoretic techniques or with the use of calculational devices that have been formulated to speed the numerical evaluation of quantum mechanical density matrices in one dimension, a problem that is mathematically equivalent to the calculation of transfer matrices in two dimensions.

We start with the Ginzburg-Landau-Wilson effective Hamiltonian of an $O(1)$ system on a lattice in three dimensions. If $s_{i}$ is the value of the continuous spin variable at the $i^{\text {th }}$ lattice site, then this effective Hamiltonian has the form

$$
\frac{1}{2} \sum_{i, j}^{\prime}\left(s_{i}-s_{j}\right)^{2}+\sum_{i}\left[\frac{r}{2} s_{i}^{2}+\frac{u}{4} s_{i}^{4}\right]
$$

The first sum in Eq. (1) is over nearest neighbor pairs. High order perturbation theory coupled with the Renormalization Group has been shown to yield very accurate results for the three-dimensional critical behavior of the system described by the effective Hamiltonian (11).

In two dimensions, the Ising model, which is based on fixed length spins, has been successfully investigated with the use of a variety of techniques. An important subset of the approaches is based on an analysis of the transfer matrix coupling the state in $2^{N}$-dimensional space of a column of spins to the state of a neighboring spin column 
11]. Onsager's exact solution [12] for the Ising model partition function consisted of the extraction of the largest eigenvalue of this transfer matrix. In the absence of techniques leading to exact solutions, one has recourse to other methods for the determination of the largest eigenvalue of a two-dimensional transfer matrix. In particular, there is a set of approaches developed to perform highly accurate numerical evaluation of the eigenvalues of the density matrix of a one-dimensional quantum mechanical system. The generic term for this collection of calculational devices is the Density Matrix Renormalization Group (DMRG) 14], 115]. There are cases in which the extremely high accuracy of this method has led to important results.

In the context of statistical mechanics, the DMRG has an analog in the form of the Transfer Matrix Renormalization Group (TMRG) [13]. The TMRG has been shown to lead to excellent results for the thermodynamic functions of the two dimensional Ising model [13], the $Q$-state Potts model [16], [17] and confinement effects in the presence of gravity [18], [19].

We have been able to demonstrate that this method can be applied to the evaluation of the partition function for the continuous-spin effective Hamiltonian (1). We start with the one-dimensional version of the Ginzburg-Landau-Wilson partition function

$$
\int \prod_{i} d s_{i} \exp \left[-\sum_{i}\left(\frac{1}{2}\left(s_{i}-s_{i+1}\right)^{2}+\frac{r}{2} s_{i}^{2}+\frac{u}{4} s_{i}^{4}\right)\right]
$$

This partition function can also be written as the inner product of a sequence of transfer matrices $T\left(s_{i}, s_{i+1}\right)$, where

$$
T\left(s_{i}, s_{j}\right)=\exp \left[-\frac{1}{2}\left(s_{i}-s_{j}\right)^{2}-\frac{r}{4}\left(s_{i}^{2}+s_{j}^{2}\right)-\frac{u}{8}\left(s_{i}^{4}+s_{j}^{4}\right)\right]
$$

Eigenfunctions, $\psi_{k}(s)$, of this transfer matrix are defined by the equation

$$
\int d s^{\prime} T\left(s, s^{\prime}\right) \psi_{k}\left(s^{\prime}\right)=\lambda_{k} \psi_{k}(s)
$$

We will list the eigenvalues in order of magnitude. The largest eigenvalue will be $\lambda_{0}$, the next largest $\lambda_{1}$ and so on. Given that the transfer matrix is real and symmetric, the eigenfunctions can also be written as real, normalized and orthogonal, in that

$$
\int_{-\infty}^{\infty} \psi_{k}(s) \psi_{l}(s) d s=\delta_{k, l}
$$

The one-dimensional transfer matrix is then written as

$$
T\left(s, s^{\prime}\right)=\sum_{k} \psi_{k}(s) \lambda_{k} \psi_{k}\left(s^{\prime}\right)
$$

Given the orthnormality of the eigenfunctions, the one-dimensional partition function has the form

$$
T^{N}\left(s, s^{\prime}\right)=\sum_{k} \psi_{k}(s) \lambda_{k}^{N} \psi_{k}\left(s^{\prime}\right)
$$

and is, thus dominated by the contribution associated with the largest eigenvalue, $\lambda_{0}$, of the transfer matrix.

Now, the $d$-dimensional Ising model can be written in terms of the eigenfunctions and eigenvalues of the onedimensional Ising model by associating neighboring pairs of spin variables in terms of a "bond" transfer matrix, $T_{b}\left(s_{i}, s_{j}\right)$, having the form

$$
\begin{aligned}
T_{b}\left(s_{i}, s_{j}\right) & =\exp \left[-\frac{1}{2}\left(s_{i}-s_{j}\right)^{2}-\frac{r}{4 d}\left(s_{i}^{2}+s_{j}^{2}\right)-\frac{u}{8 d}\left(s_{i}^{4}+s_{j}^{4}\right)\right] \\
& =\sum_{k} \psi_{k}\left(s_{i}\right) \lambda_{k} \psi_{k}\left(s_{j}\right)
\end{aligned}
$$

The partition function is then a sum over bond indices, $k$, while vertices contain integrations over the spin variable $s$ of combinations of transfer matrix eigenfunctions:

$$
\int_{-\infty}^{\infty}\left(\prod_{l=1}^{2 d} \psi_{k_{l}}(s)\right) d s
$$


While the eigenfunctions, $\psi_{k}(s)$ are orthonormal, there is no reason to believe that this will lead to significant restrictions over the allowed indices in the integration of a product of $2 d$ of the $\psi_{k}$ 's when $d>2$. One important simplification arises from the fact that a $\psi_{k}(s)$ will be either even or odd in the spin variable $s$. In fact, the largest eigenvalue is associated with an even-parity eigenfunction and the next largest with an odd-parity eigenfunction.

The partition function of the two-dimensional continuous-spin variable version of the $O(1)$ model was calculated with the use of the formulation above in terms of transfer matrix eigenfunctions, the basis set being truncated at the eigenfunctions having the largest few eigenvalues. The fourth order coupling constant, $u$, in (11) was chosen to be equal to 0.23 , guided by a best fit between the Ginzburg-Landau Wilon effective Hamiltonian and order parameter distributions in three dimensions as obtained by simulation [20]. These eigenfunctions were used to construct the basis set of the two-dimensional transfer matrix connecting one row of continuous spins to the next. This two-dimensional transfer matrix was then reduced with the use of an adaptation of the TMRG. The free energy is extracted from the largest eigenvalue of the transfer matrix via the connection $\mathcal{F}=-k_{B} T \ln \lambda_{0}$. Because we are interested in the behavior of the partition function in the immediate vicinity of the critical point, temperature-dependent factors multiplying the log of the largest eigenvalue are set equal to a constant.

This version of the free energy leads immediately to the specific heat through differentiation with respect to the "bare" reduced temperature, $r$. It is also possible to introduce a symmetry-breaking field, $h$, appearing in the effective Hamiltonian through the term $-\sum_{i} h s_{i}$. The remanent magnetization and the isothermal susceptibility can be calculated from the free energy by taking, respectively, first and second derivatives with respect to the symmetrybreaking field.

The spontaneous magnetization of the system can be calculated from the spin operator $s_{2}$

$$
M=\left\langle\Psi_{0}\left|s_{2}\right| \Psi_{0}\right\rangle
$$

where $\Psi_{0}$ is the eigenfunction of the transfer matrix with the largest eigenvalue and the spin operator $s_{2}$ is the Ising spin operator. We plot the value of (10) on a log-log grid to illuminate the power-law behavior (see Figure 1). The value of the critical exponent $\beta$ is calculated to be $0.1229 \pm 0.0006$ which is consistent with the exact value for the $d=2$ Ising model of $1 / 8$ [11].

Differentiating the free energy twice with respect to the reduced temperature $r$, we obtain the specific heat. We display our results for this quantity as a function of $r$ in Figure 2. Further analysis of these data reveal a logarithmic dependency both above and below the critical temperature, in other words $\alpha=\alpha \prime=0$, as expected from the Ising model.

We have also examined the ratio of specific heat amplitudes $A_{+} / A_{-}$where we fit to the function

$$
C_{ \pm}=A_{ \pm} \ln \left|r-r_{c}\right|+B_{ \pm}
$$

For an $O(1)$ system in two dimensions, this value is expected to be unity. The value calculated from the data is 0.94 .

As mentioned in the previous section, by adding a symmetry-breaking term $-\sum_{i} h s_{i}$, we are able to take derivatives with respect to $h$ to obtain the spontaneous magnetization (independently from the $\left\langle\Psi_{0}\left|s_{2}\right| \Psi_{0}\right\rangle$ method) and the magnetic susceptibility $\chi_{T}$. Determination of the magnetization using this approach gives $\beta=0.1230 \pm 0.0009$.

The calculation of $\chi_{T}$ requires special consideration as it is dependent on the size of the "grid" $\Delta h$ used for the numerical differentiation. Best results for the critical exponent above and below the critical temperature are: $\gamma=1.74 \pm 0.01$ and $\gamma \prime=1.33 \pm 0.03$. The exponents do appear to converge to the expected value of $7 / 4$ as the grid size $d h$ is reduced (see Figure 3 ).

Finally we comment on the validity of the assumption that the retention of a small number of states in the eigenfunction expansion of the transfer matrix (6) suffices to ensure an accurate calculation of critical point properties. The previous results were obtained by using only two states in the expansion (6), i.e. $n_{s}=2$. To empirically investigate the inclusion of more states, the calculation of the specific heat was repeated with $n_{s}=4$. The results are effectively the same within error bounds $-\alpha=\alpha \prime=0$ and the amplitude ratio is 0.95 .

There is another perspective from which to explore the effect of truncating the Ginzburg-Landau basis. The lowest order effect of including higher states can be realized by allowing a single bond in the lattice to be represented by the full sum of states in the expansion. We will refer to this as an "enhanced" bond. All other bonds are expanded on only the first two $\left(n_{s}=2\right)$ eigenstates. We can then observe the effect of this enhancement on the free energy density as we increase $n_{s}$. Examining Figure 1 it is clear that the effect of enhancement is negligible above $n_{s}=4$.

$\dagger \quad$ Presently at PCS, 11859 Wilshire Blvd., Suite 600, Los Angeles, CA 90025. 
[1] G. A. Baker, Jr., B. G. Nickel, M. S. Green, et al., Physical Review Letters 36, 1351 (1976).

[2] J. C. le Guillou and J. Zinn-Justin, Physical Review Letters 39, 95 (1977).

[3] W. Lenz, Phys. Zeitschrift 21, 613 (1920).

[4] E. Ising, Zeitschrift f. Physik 31, 253 (1925).

[5] M. N. Barber and M. E. Fisher, Annals of Physics 77, 1 (1973).

[6] M. N. Barber and M. E. Fisher, Physical Review A (General Physics) 8, 1124 (1973).

[7] S. Singh and R. K. Pathria, Physical Review A (General Physics) 30, 442 (1984).

[8] S. Singh and R. K. Pathria, Physics Letters A 118, 131 (1986).

[9] D. O'Connor and C. R. Stephens, International Journal of Modern Physics A 9, 2805 (1994).

[10] D. O'Connor, C. R. Stephens, and A. J. Bray, Journal of Statistical Physics 87, 273 (1997).

[11] R. J. Baxter, Exactly solved models in statistical mechanics (Academic Press, London ; New York, 1982).

[12] L. Onsager Phys. Rev. 65, 117 (1944).

[13] T. Nishino, J. Phys. Soc. Jpn. 64, 3598 (1995).

[14] S.R. White, Phys. Rev. Lett. 69, 2863 (1992).

[15] S.R. White, Phys. Rev. B 48, 10345 (1993).

[16] F. Iglói and E. Carlon, Phys. Rev. B 59, 3783 (1999).

[17] E. Carlon and F. Iglói, Phys. Rev. B 57, 7877 (1998).

[18] E. Carlon and A. Drzewínski, Phys. Rev. E 57, 2626 (1998).

[19] E. Carlon and A. Drzewínski, Phys. Rev. Lett. 79, 1591 (1997)

[20] J. Rudnick, W. Lay, and D. Jasnow, Phys. Rev. E 58, 2902 (1998).

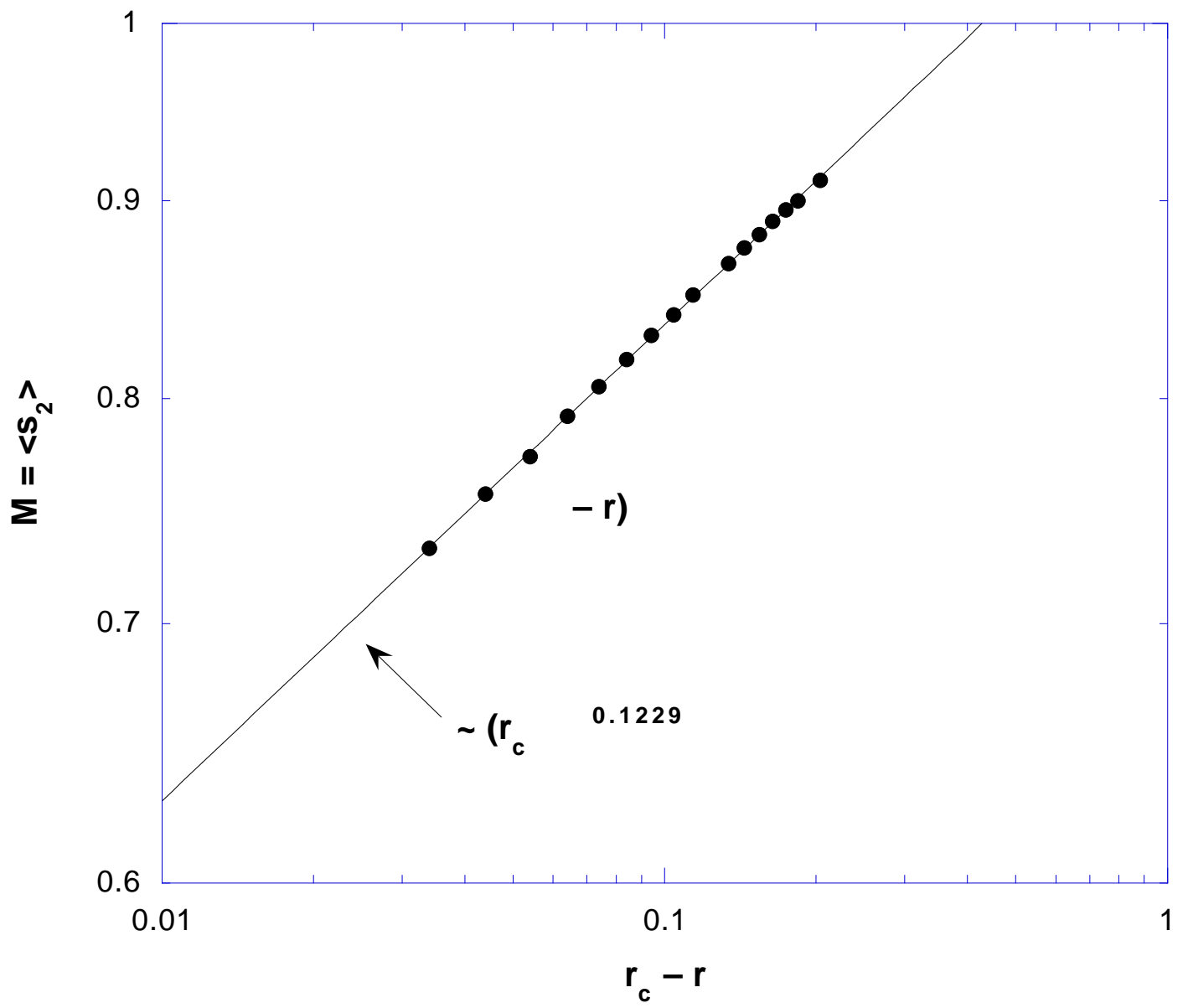

FIG. 1. The spontaneous magnetization (calculated as the expectation value in the state $\left.\left|\Psi_{0}\right\rangle\right)$ as a function of $\left(r_{c}-r\right)$. 


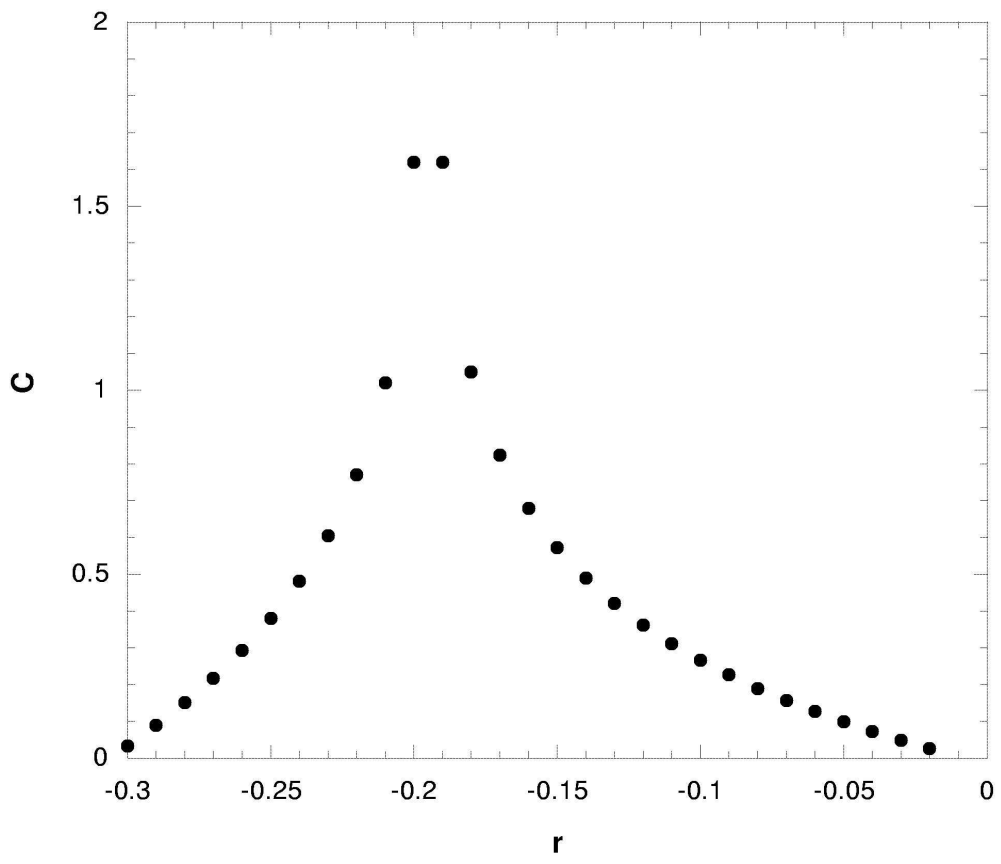

FIG. 2. The specific heat as a function of $r$. 


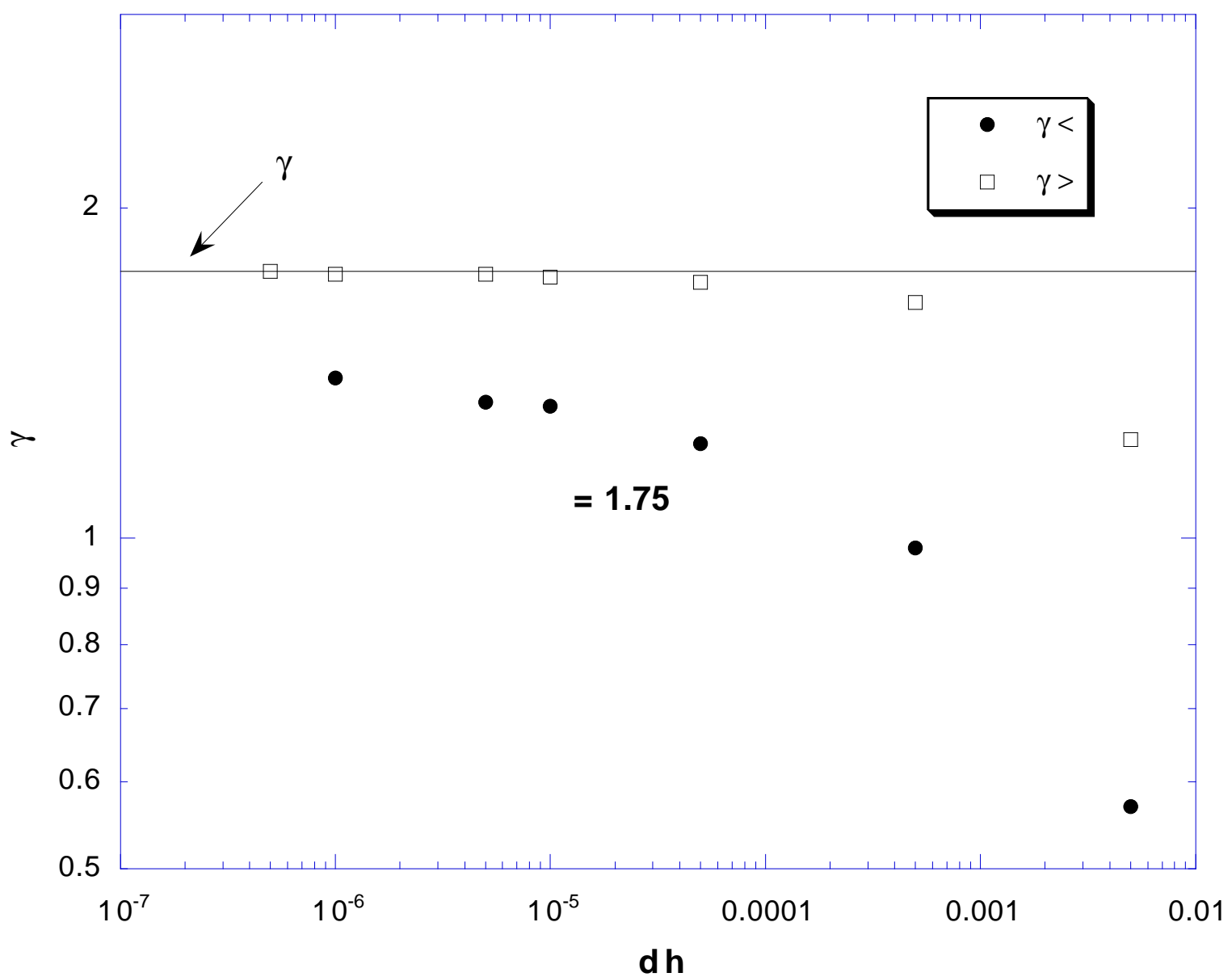

FIG. 3. The susceptibility exponent $\gamma$ above and below the critical temperature as a function of the grid spacing $d h$. The horizontal line denotes the $2 D$ Ising exponent of $\gamma=1.75$. 


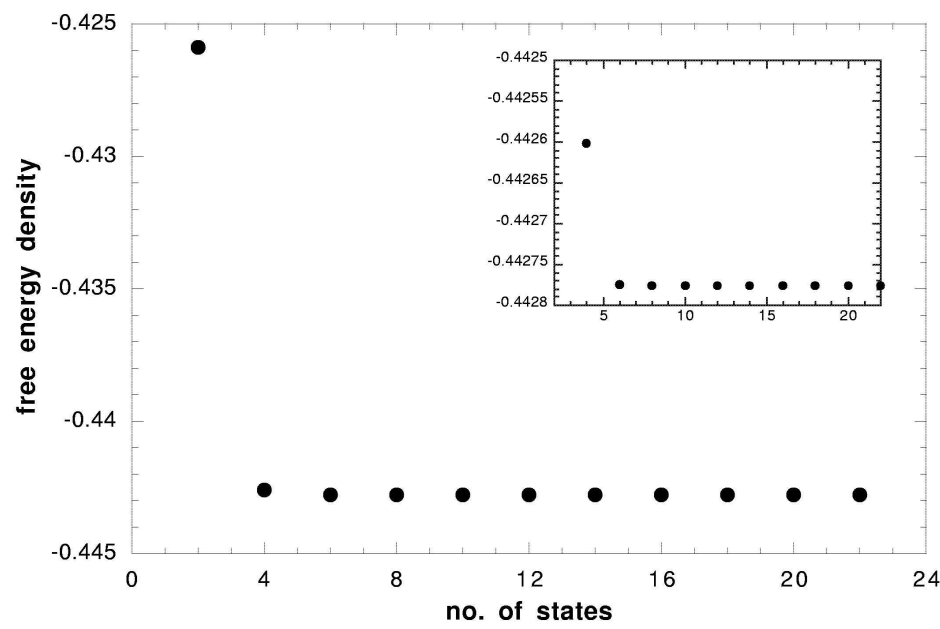

FIG. 4. The free energy of a chain with a single enhanced bond as a function of $n_{s}$ (i.e. the number of states kept in the expansion of the Ginzburg-Landau transfer matrix). The inset shows the detail of $n_{s}=4$ and higher. 